\title{
Prospective Comparison of Saliva and Nasopharyngeal Swab Sampling for Mass Screening for COVID-19
}

OPEN ACCESS

Edited by:

Catherine Ropert,

Federal University of Minas

Gerais, Brazil

Reviewed by:

Nitya Singh,

University of Florida, United States

Guy Thwaites,

University of Oxford, United Kingdom

*Correspondence:

Mathieu Nacher

mathieu.nacher66@gmail.com

Specialty section:

This article was submitted to Infectious Diseases - Surveillance,

Prevention and Treatment, a section of the journal

Frontiers in Medicine

Received: 25 October 2020 Accepted: 03 February 2021 Published: 23 February 2021

Citation:

Nacher M, Mergeay-Fabre M, Blanchet D, Benoit O, Pozl T,

Mesphoule P, Sainte-Rose V,

Vialette $V$, Toulet B, Moua A, Saout M,

Simon S, Guidarelli M, Galindo M,

Biche B, Faurous W, Chaizemartin L,

Fahrasmane $A$, Rochemont $D$,

Vignier $N$, Vabret $A$ and Demar $M$ (2021) Prospective Comparison of Saliva and Nasopharyngeal Swab

Sampling for Mass Screening for COVID-19. Front. Med. 8:621160

doi: 10.3389/fmed.2021.621160

\begin{abstract}
Mathieu Nacher ${ }^{1,2 *}$, Mayka Mergeay-Fabre ${ }^{1}$, Denis Blanchet ${ }^{3}$, Orelie Benoit ${ }^{4}$, Tristan Pozl ${ }^{4}$, Pauline Mesphoule ${ }^{4}$, Vincent Sainte-Rose ${ }^{3}$, Véronique Vialette ${ }^{3}$, Bruno Toulet ${ }^{3}$, Aurélie Moua ${ }^{3}$, Mona Saout ${ }^{5}$, Stéphane Simon ${ }^{3}$, Manon Guidarelli ${ }^{1}$, Muriel Galindo ${ }^{1}$, Barbara Biche ${ }^{1}$, William Faurous ${ }^{1}$, Laurie Chaizemartin ${ }^{6}$, Aniza Fahrasmane ${ }^{1}$, Devi Rochemont ${ }^{1}$, Nicolas Vignier ${ }^{1}$, Astrid Vabret $^{7}$ and Magalie Demar ${ }^{3,5}$
\end{abstract}

${ }^{1}$ Centre d'Investigation Clinique (CIC) Institut National de la Santé et de la Recherche Médicale (INSERM) 1424, Centre Hospitalier de Cayenne Andrée Rosemon, Cayenne, French Guiana, ${ }^{2}$ Département Formation Recherche (DFR) Santé, Université de Guyane, Cayenne, French Guiana, ${ }^{3}$ Laboratoire, Centre Hospitalier de Cayenne Andrée Rosemon, Cayenne, French Guiana, ${ }^{4}$ Centre de Ressources Biologiques Amazonie, Centre Hospitalier de Cayenne Andrée Rosemon, Cayenne, French Guiana, ${ }^{5}$ Unité Mixte de Recherche Tropical Biome and Immuno-Pathology (TBIP), Université de Guyane, Cayenne, French Guiana, ${ }^{6}$ Centre Délocalisé de Prévention et Soins de Maripasoula, Maripasoula, French Guiana, ${ }^{7}$ Service de Virologie, CHU de Caen, Caen, France

Current testing for COVID-19 relies on reverse-transcriptase polymerase chain reaction from a nasopharyngeal swab specimen. Saliva samples have advantages regarding ease and painlessness of collection, which does not require trained staff and may allow self-sampling. We enrolled 776 persons at various field-testing sites and collected nasopharyngeal and pooled saliva samples. One hundred sixty two had a positive COVID-19 RT-PCR, 61\% were mildly symptomatic and 39\% asymptomatic. The sensitivity of RT-PCR on saliva samples vs. nasopharygeal swabs varied depending on the patient groups considered or on Ct thresholds. There were $10(6.2 \%)$ patients with a positive saliva sample and a negative nasopharyngeal swab, all of whom had Ct values $<25$ for three genes. For symptomatic patients for whom the interval between symptoms onset and sampling was $<10$ days sensitivity was $77 \%$ but when excluding persons with isolated $\mathrm{N}$ gene positivity (54/162), sensitivity was $90 \%$. In asymptomatic patients, the sensitivity was only $24 \%$. When we looked at patients with Cts $<30$, sensitivity was 83 or 88.9\% when considering two genes. The relatively good performance for patients with low Cts suggests that Saliva testing could be a useful and acceptable tool to identify infectious persons in mass screening contexts, a strategically important task for contact tracing and isolation in the community.

Keywords: COVID-19, saliva, sensitivity, PCR, nasopharyngeal

\section{INTRODUCTION}

Current testing for COVID-19 relies on reverse-transcriptase polymerase chain reaction (RT-PCR) from a nasopharyngeal swab specimen (1). Nasopharyngeal sampling requires human resources and training, personal protective equipment and swabs, and time, generating testing bottlenecks and potential exposure to transmission at crowded testing sites. Moreover, the unpleasantness of the procedure and the long waiting delays for swab collection and results may dissuade some persons 
to get tested or to repeat tests when they are negative. There is an urgent need for innovative testing strategies to rapidly identify cases, reduce waiting delays, and facilitate mass screening. Saliva samples have advantages regarding ease and painlessness of collection, which does not require trained staff and may allow self-sampling. The comparison of real time PCR results on salivary and nasopharyngeal samples has shown discrepancies between studies, with most finding greater sensitivity and lower RT-PCR Cts in nasopharyngeal swab samples (2-4) whereas others found greater sensitivity in saliva samples $(5,6)$. The sources of variation may have been the study population (hospitalized patients vs. screening of contacts or mildly symptomatic patients), saliva collection techniques and timing, conditioning and delays in processing raw saliva samples, or differences in the RT-PCR techniques used.

French Guiana is an Overseas French territory between Brazil and Suriname. Although it has a French Health System, it is isolated and its limited hospital capacity is vulnerable to the COVID 19 epidemic surge. As the epidemic peaked in July 2020, intense efforts were undertaken to expand hospital and ICU capacity, to continue contact tracing and offer a place to quarantine for patients that were unable to isolate themselves at home, and to expand COVID-19 testing and reduce testing bottlenecks at the public and private laboratories on the territory and the ensuing renouncement to get tested. We here report the first prospective study of the performance of saliva testing compared to nasopharyngeal swabs in a field context of mass screening in French Guiana.

\section{METHODS}

\section{Context}

This French territory neighboring Amapa state in Brazil has been highly impacted by COVID-19 with $3.2 \%$ of the population having had a confirmed infection, notably among the poorest populations (7). In this context, testing and tracking were implemented throughout the epidemic, testing tents and mobile testing teams including the remote health centers, the Red Cross, Médecins du Monde, and the reinforcements from the Réserve Sanitaire were coordinated by the regional health agency to investigate around clusters of cases. The testing efforts for this small population peaked to nearly $0.5 \%$ of the population screened in a day.

\section{Study Conduct}

Between July 22th and September 10th, we prospectively enrolled consecutive, persons aged 3 years or more with mild symptoms suggestive of COVID-19 and asymptomatic persons with a testing indication at various testing sites and mobile testing brigades in French Guiana reaching remote sites up to $240 \mathrm{~km}$ in the Amazonian Forest. During screening missions, mobile teams, consisting of Healthcare personnel (doctors, nurses) were coordinated by the Health Regional Agency of French Guiana, targeting villages, neighborhoods, where the virus was circulating collected persons often out of doors or in health centers. These mobile teams were made up of staff from the Red Cross, Médecins du Monde, the Cayenne hospital PASS, the Maripa Soula health center, and the health reserve. Team travel was coordinated and decided by the health regional agency of French Guiana each week during a weekly update and was guided the knowledge of current clusters of cases which triggered screening campaigns in the concerned neighborhoods-urban or rural, and usually socially disadvantaged; in addition, patients requiring hospitalization for non-COVID reasons (for example a fractured limb) were screened to rule out infectiousness; during the peak of the epidemic drive through testing services were also deployed to offer testing to any person requesting a test. Inclusion criteria were: males or females with an indication to perform a COVID diagnostic test (symptomatology, contact case, systematic screening, etc.), aged at least 3 years old. Noninclusion criteria were refusal of the patient or his/her legal representative, person taking treatments that reduce salivary volume (anticholinergic activity), impossibility of carrying out the Nasopharyngeal swab, and persons under guardianship or curatorship, or placed under protective measures. All study participants were enrolled and sampled in accordance with the protocol. An investigator explained the objectives of the study and obtain the oral consent of the patient or his/her legal representative. The form was completed by the investigator or a person delegated by the investigator. The trained nurse present during the testing mission performed the nasopharyngeal swab and collected the salivary sputum sample in a urine container. A trained agent carried out a short questionnaire. At the end of each day, all completed forms and samples were sent to Cayenne hospital and stored at $4^{\circ} \mathrm{C}$ before analysis. Samples and participant information were non-individually identifiable and collected with a unique identifying number.

\section{Laboratory Analysis}

The same technique was used for the two samples throughout the study: the QIAsymphony and GeneFinder kit, a Real-time PCR assay. GeneFinder ${ }^{\mathrm{TM}}$ COVID-19 detects SARS-CoV-2 by amplification of RdRp gene, $\mathrm{E}$ gene, and $\mathrm{N}$ gene according to WHO's recommended protocol. Viral nucleic acid was extracted by using the QIAamp DSP viral kit on the QIAsymphony RGQ, an integrated fully automated nucleic acid extraction (chemical lysis and paramagnetic bead binding) and sample preparation platform (Qiagen GmbH, Germany). The real-time PCR assays for SARS Cov2 were performed with an Applied 7500 cycler (Thermofisher) with the Genefinder kit (Ellitech group) that could detect the $\mathrm{N}$ gene, RdRp and $\mathrm{E}$ gene, which is not specific to COVID-19. As the Nucleic acid extraction methods could affect the results of viral nucleic acid amplification tests, we treated the couple saliva-nasopharyngeal specimens with the same method and most of the time in the same series, the eluates were obtained from $200 \mu \mathrm{l}$ of specimens ( $300 \mu \mathrm{L}-100 \mu \mathrm{L}$ dead volume). The remainder of each sample was divided into paired aliquots kept in a biorepository for further studies evaluating new screening tools.

\section{Statistical Analysis}

Statistical analysis was performed using STATA ${ }^{\circledR} 16$ (Stata corporation, College Station, Texas, USA). Cross tabulations considering different subgroups was performed. We considered the RdRp and $\mathrm{N}$ genes, specific for SARS-Cov2, to calculate 
different $\mathrm{Ct}$ categories. The raw data can be accessed at https:// doi.org/10.7910/DVN/KPLJ9A.

\section{Ethical}

The protocol received ethical approval from the Comite de Protection des Personnes under the number 2020-A0200930/SI:20.07.07.54744.

\section{RESULTS}

We included 776 patients between July 22th and September 10th. The sex ratio $(\mathrm{M} / \mathrm{F})$ was 1.6 , the mean age was 40 (standard deviation $=16.8)$. Overall, $61 \%$ were mildly symptomatic and $39 \%$ were asymptomatic. For symptomatic patients, $84 \%$ had a symptoms onset $<10$ days, and $4 \%$ were hospitalized within 2 weeks after inclusion.

\section{Patients With Positive RT-PCR}

The crude analysis showed that 152 had a positive RT-PCR on the nasopharyngeal sample and 86 had a positive RT-PCR on the saliva sample; 76 persons had both a positive Nasopharyngeal and Saliva RT-PCR result, while 76 had a positive nasopharyngeal RTPCR but a negative saliva RT-PCR; Finally, 10 patients (6.2\%) had a negative Nasopharyngeal RT-PCR but a positive saliva RT-PCR

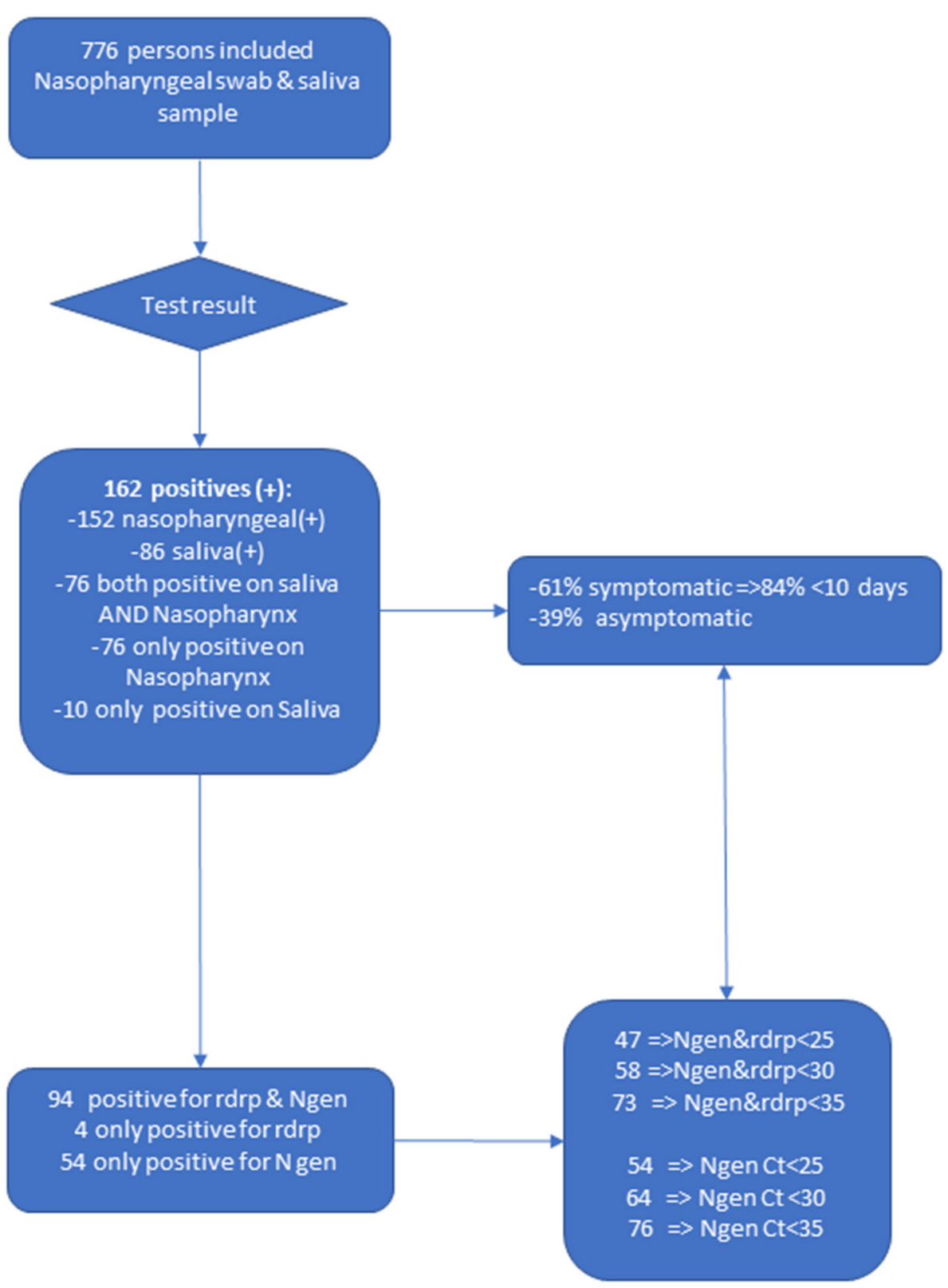

FIGURE 1 | Flow chart of the Covisal study. 

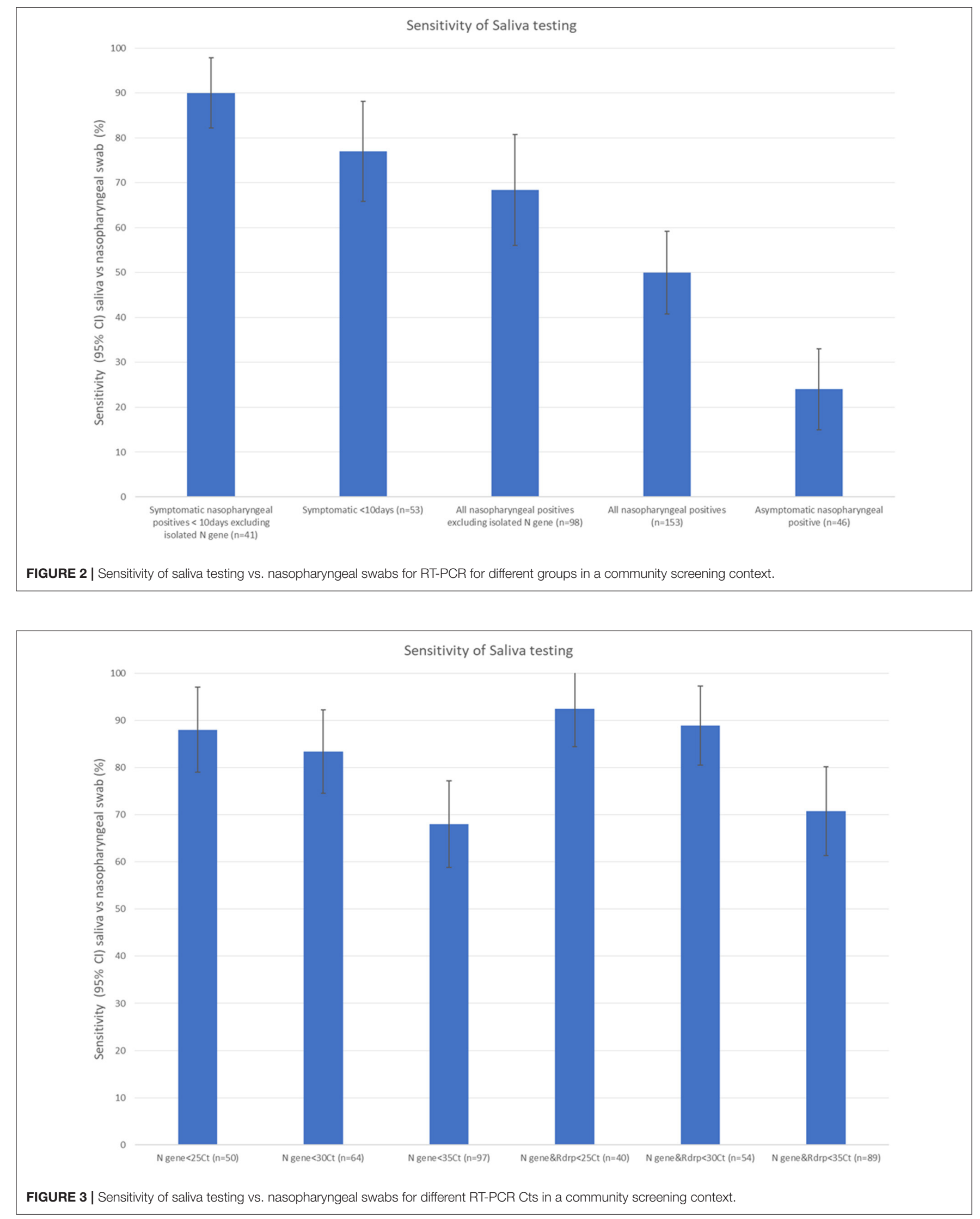


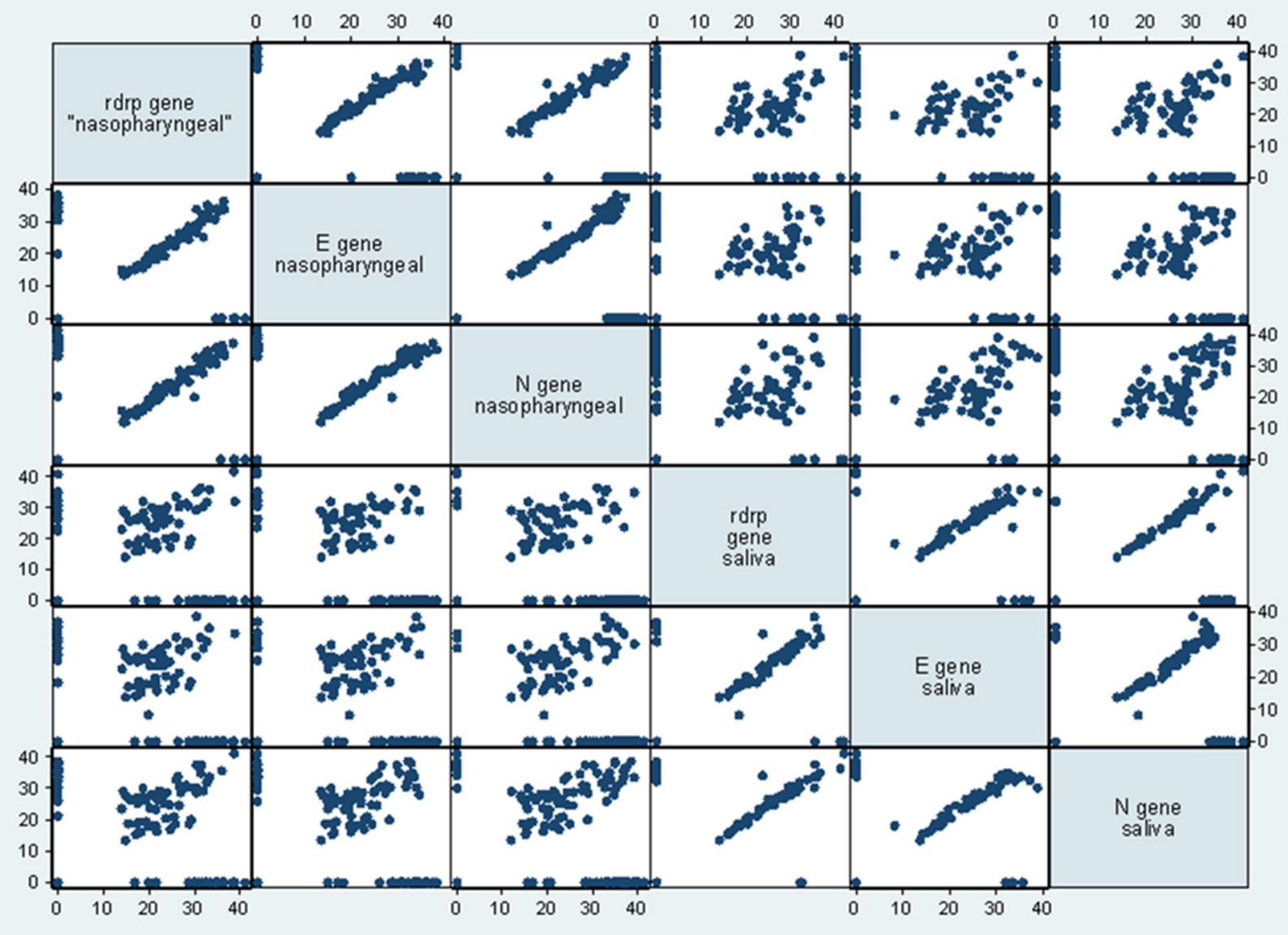

FIGURE 4 | Scatterplot matrix for the Ct of different genes in the nasopharyngeal and saliva samples. There was a "fanning" pattern with greater dispersion at higher $\mathrm{Ct}$ values for different genes in the nasopharyngeal and saliva samples.

(Figure 1). In total 162 (20.9\%) of patients had a positive result on either the Nasopharyngeal or the saliva sample.

\section{Sensitivity, Symptoms, and Ct Values}

The sensitivity of RT-PCR on saliva samples vs. nasopharygeal swabs varied depending on the patient groups considered (Figure 2) or on Ct thresholds (Figure 3). When considering all patients with at least one gene amplification-irrespective of delays, symptoms, or Cts, sensitivity was low (50\%); For symptomatic patients with an interval between symptoms onset and sampling under 10 days sensitivity was $77 \%$; however, when excluding persons with isolated $\mathrm{N}$ gene positivity (54/162) from this subgroup, sensitivity was $90 \%$.For asymptomatic patients, the sensitivity was only $24 \%$, the lowest of all studied groups (Figure 2).

Recent studies have argued that transmission potential estimated by the capacity to infect cell cultures- was restrained to those with low Cts $(8,9)$, a proxy for high viral load. When we looked at patients with Cts $<30$, sensitivity was 83 or $88.9 \%$ when considering two genes. Among the 10 patients with a positive saliva sample and a negative nasopharyngeal swab, all had Ct values $<25$. Figure 4 shows increasing dispersion for the higher
Ct values of the nasopharyngeal vs. saliva sample scatterplots for the different genes amplified by RT PCR emphasizing the greater discordance between samples among patients with lower viral loads.

\section{DISCUSSION}

Contrarily to two studies suggesting a greater positivity rate for saliva $(5,6)$, we observed that saliva testing was less sensitive than nasopharyngeal swabs. Whereas, most studies were hospitalbased collecting saliva in the early morning before mouth rinsing and breakfast, our study was a screening study that was performed in difficult field conditions targeting hard to reach populations after breakfast and teeth brushing, moreover out of doors in a tropical context. These realistic conditions were however also a limitation because of the heterogeneity of inclusion sites. Since the main objective was to compute sensitivity, in order to shorten the time allocated to each inclusion, there was limited clinical/epidemiology data from tested individuals and no data on possible repeated testing. The study started after the epidemic peak and hence inclusion of 
positive patients became increasingly difficult, and the number of positives was hence insufficient to conduct stratified analyses on subgroups, and particularly for asymptomatic persons with positive RT-PCR who may pool active infections and residual shedding but no clear time frame that could allow to disentangle the two. The poor sensitivity on asymptomatic positive nasopharyngeal swabs was thus presumably also linked to the inclusion of non-infectious patients in the denominator. A third of positives only had a positive $\mathrm{N}$ gene, the RdRp and E gene being negative. Based on the empirical experience of the laboratory, such patients were considered to be at later stages of the infection.

The relatively good performance for patients with low Cts suggests that Saliva testing could be a useful and acceptable tool to identify infectious persons in mass screening contexts, a strategically important task for contact tracing and isolation in the community. With the considerable testing bottlenecks, alleviating the workload and shortening the sample collection time would be improvements that could reduce waiting times to get tested and human-resource costs. The sensitivity saliva samples for asymptomatic persons seemed insufficient but without any temporal indication about the onset of infection, it should be further studied by $\mathrm{Ct}$ values with a larger sample size. In view of the present results the French Health authorities have officially declared that saliva testing may be used on symptomatic patients only when nasopharyngeal tests cannot be used (10).

\section{DATA AVAILABILITY STATEMENT}

The raw data supporting the conclusions of this article will be made available by the authors, without undue reservation.

\section{REFERENCES}

1. Tang YW, Schmitz JE, Persing DH, Stratton CW. Laboratory diagnosis of COVID-19: current issues and challenges. J Clin Microbiol. (2020) 58:e0051220. doi: 10.1128/JCM.00512-20

2. Chen JHK, Yip CCY, Poon RWS, Chan KH, Cheng VCC, Hung IFN, et al. Evaluating the use of posterior oropharyngeal saliva in a point-of-care assay for the detection of SARS-CoV-2. Emerg Microbes Infect. (2020) 9:13569. doi: $10.1080 / 22221751.2020 .1775133$

3. Migueres M, Mengelle C, Dimeglio C, Didier A, Alvarez M, Delobel $\mathrm{P}$, et al. Saliva sampling for diagnosing SARS-CoV-2 infections in symptomatic patients and asymptomatic carriers. J Clin Virol. (2020) 130:104580. doi: 10.1016/j.jcv.2020.104580

4. Williams E, Bond K, Zhang B, Putland M, Williamson DA. Saliva as a noninvasive specimen for detection of SARS-CoV-2. J Clin Microbiol. (2020) 58:e00776-20. doi: 10.1128/JCM.00776-20

5. Rao M, Rashid FA, Sabri FS, Jamil NN, Zain R, Hashim R, et al. Comparing nasopharyngeal swab and early morning saliva for the identification of SARS-CoV-2. Clin Infect Dis. (2020). doi: 10.1093/cid/ciaa1156. [Epub ahead of print].

6. Wyllie AL, Fournier J, Casanovas-Massana A, Campbell M, Tokuyama M, Vijayakumar P, et al. Saliva or nasopharyngeal swab specimens for detection of SARS-CoV-2. N Engl J Med. (2020) 383:1283-6. doi: 10.1056/NEJMc2016359

7. Point Epidémio Régional hebdomadaire "Spécial COVID-19". Available online at: https://www.guyane.ars.sante.fr/point-epidemio-regional-hebdomadairespecial-covid-19 (accessed September 9, 2020).

\section{ETHICS STATEMENT}

The studies involving human participants were reviewed and approved by Comité de Protection des Personnes. Written informed consent to participate in this study was provided by the participants' legal guardian/next of kin.

\section{AUTHOR CONTRIBUTIONS}

MN and MD: conception. MM-F, DB, OB, TP, PM, VS-R, VV, BT, AM, MS, SS, MGu, MGa, BB, WF, LC, AF, DR, NV, AV, and MD: investigation. $\mathrm{MN}$ : analysis and first draft writing. MN, MM-F, and MD: review and editing. All authors contributed to the article and approved the submitted version.

\section{FUNDING}

This project was funded by the Ministry of Higher Education and Research. The funder had no influence on the content of the research.

\section{ACKNOWLEDGMENTS}

We wish to express our gratitude to the directions and personnel of the Agence Régionale de Santé de Guyane, Centre Hospitalier de Cayenne, the Croix Rouge Française, Médecins du Monde, the Permanence d'Accès aux Soins de Santé, the Réserve Sanitaire, Santé Publique France, the Centres Délocalisés de Prévention et de Soins and numerous Health mediators, and REACTing.

8. Cevik M, Tate M, Lloyd O, Maraolo AE, Schafers J, Ho A. SARS-CoV-2, SARS-CoV-1 and MERS-CoV viral load dynamics, duration of viral shedding and infectiousness: a living systematic review and meta-analysis. medRxiv. (2020). doi: 10.1101/2020.07.25.20162107

9. Rhee C, Kanjilal S, Baker M, Klompas M. Duration of SARS-CoV2 infectivity: when is it safe to discontinue isolation? Clin Infect Dis. (2020). doi: 10.1093/cid/ciaa1249. [Epub ahead of print].

10. COVID-19: les tests salivaires peuvent compléter les tests nasopharyngés chez les personnes symptomatiques. Haute Autorité de Santé. Available online at: https://www.has-sante.fr/jcms/p_3202317/fr/covid-19-les-testssalivaires-peuvent-completer-les-tests-nasopharynges-chez-les-personnessymptomatiques (accessed September 18, 2020).

Conflict of Interest: The authors declare that the research was conducted in the absence of any commercial or financial relationships that could be construed as a potential conflict of interest.

Copyright (c) 2021 Nacher, Mergeay-Fabre, Blanchet, Benoit, Pozl, Mesphoule, Sainte-Rose, Vialette, Toulet, Moua, Saout, Simon, Guidarelli, Galindo, Biche, Faurous, Chaizemartin, Fahrasmane, Rochemont, Vignier, Vabret and Demar. This is an open-access article distributed under the terms of the Creative Commons Attribution License (CC BY). The use, distribution or reproduction in other forums is permitted, provided the original author(s) and the copyright owner(s) are credited and that the original publication in this journal is cited, in accordance with accepted academic practice. No use, distribution or reproduction is permitted which does not comply with these terms. 